\title{
Study On The PATH Tracking AND Positioning Method OF WHEELEd Mobile ROBOT
}

\author{
Cheng Jing and Cheng Wushan \\ College of Mechanical Engineering, Shanghai University of Engineering Science, \\ Shanghai 201620, China
}

\begin{abstract}
As a kind of wheeled mobile robot used in intelligent logistics system, AGV is mainly used for automatic material transportation, the precise positioning and path tracking is the assu-rance of accurate material transportation. In this article, the laser coordinate positioning technology is used to realize accurate positioning for AGV, a new method of target reference point selection is put forward, and path tracking is implemented in combination with the kinematics model of single steering wheel AGV, the objective function that $A G V$ successfully reaches the destination accurately according to the preset trajectory is completed finally. The study is in trial stage, and obtains good operation effectiveness.
\end{abstract}

\section{KEY WORDS}

Wheeled Mobile Robot, Laser Positioning, Path Tracking, Kinematics Model.

\section{INTRODUCTION}

Wheeled mobile robot of Logistics system is also known as automatic guided vehicle (AGV), mainly used for the transportation of all kinds of material in warehouse, it is an important equipment in logistics system of modern manufacturing enterprise, it provides an important guarantee for integration, flexible and efficient operation of the system. The accurate positioning of AGV, combined with its effective track to the desired trajectory, can greatly improve the accuracy of material transportation in the three-dimensional warehouse.

At present, according to the particularity of working environment, a lot of positioning methods are available; they are classified into the orientation of inertial and beacon, according to the positioning based on sensor information types [1]. Independence of inertial positioning is strong, but the positioning error has a cumulative effect, it should be periodically revised [2]; Laser positioning and visual positioning are two of the most commonly techniques in beacon positioning. Visual position including image processing is greatly influenced by light conditions, such as shadow, with poor real-time performance and high system cost. Laser positioning is of high precision and good flexibility, and there is no need to deal with the ground, which is convenient for path transformation and suitable for field environment, meanwhile it is convenient to modify the motion parameters and driving paths. Laser positioning is one of the most advanced guidance in the word [3].

The laser coordinate positioning technology is used to realize accurate positioning of AGV in this paper. We can get the angle deviation to be corrected in real-time during the path tracking process, through the analysis of the kinematics model of single steering wheel AGV, and 
constantly modify the primary drive angle deviation. At the same time, on the choice of reference target a new method is adopted, in this method, target reference points move coherently with the $\mathrm{AGV}$, making that the tracking accuracy would not reduce for AGV getting close to the reference point in the movement, avoiding the redundancy of system caused by setting too much reference points. Which is different from general path tracking methods? We develop a method of locating and tracking of wheeled mobile robot, this method can meet the requirements of intelligent warehouse for AGV delivery location accuracy.

\section{LASER COORDINATE POSITIONING TECHNOLOGY}

\subsection{Classification of Laser Positioning Algorithms}

For AGV navigation module, the application of laser positioning algorithms includes the triangulation method and the three-point positioning method. The triangulation method, is known by calculating the position and orientation of AGV through the position of the reflecting plate and the angle between the AGV and them. This method requires the measurement signal with very high angular resolution and narrow beam, it is generally used in the occasion with laser positioning. The disadvantage of the three-point positioning method is that the cost of location device is high since measuring absolute distance between the receiver to the reflective beacon such as reflector column is necessary, and positioning system must have a synchronization time as a benchmark which is very complex ${ }^{[4]}$.

\subsection{Initialization of Coordinate System}

For obtaining AGV location data in the actual operation process, initialize the coordinate system before the car movement, determine the coordinate information of each laser reflector, and see them as the absolute coordinate system ${ }^{[5]}$. Since cylindrical reflector can be measured at any angle, we design reflector into a cylinder, and initialization process is as follows.

Choose the origin position of absolute coordinate system, and the position must ensure that the laser scanning head can scan more than 3 pieces of reflective pillars, and the AGV keeps still at the origin; Open laser scanning head, by scanning the distance and angel between reflective pillar and the scan head, the position information of each reflective pillar in the absolute coordinate system can be determined ${ }^{[6]}$; In the actual movement process of AGV, The coordinate information is coordinate reference point for $\mathrm{AGV}$ pose.

\subsection{Laser Positioning Principle}

For realizing the laser guidance of AGV, place a certain number of reflective pillars around fixed location in its running environment, the exact values of their coordinates are known and stored on control computer of AGV onboard ${ }^{[7]}$. Install a laser emission and detection device on the top of $\mathrm{AGV}$, when laser beam fires into reflective pillars, most of the energy is reflected and detected by photosensitive diode which is mounted near the emission source, at the same time, the angle made by reflected light and advancing direction of AGV is determined.

The AGV in action continually receives reflected laser beam from 3 (or more) known positions, and we can determine the exact location and orientation of AGV through the calculation of geometric on azimuth relative to the direction of the $\mathrm{AGV}^{[8]}$.

As shown in Figure 1, AGV has measured reflected laser beam from 3 (or more) known reference points $\left(R_{1}, R_{2}, R_{3}\right)$, their coordinates $\left(x_{1}, y_{1}\right),\left(x_{2}, y_{2}\right)$ and $\left(x_{3}, y_{3}\right)$ are known in the coordinate system xoy. 
International Journal of Computer Science \& Engineering Survey (IJCSES) Vol.6, No.3, June 2015

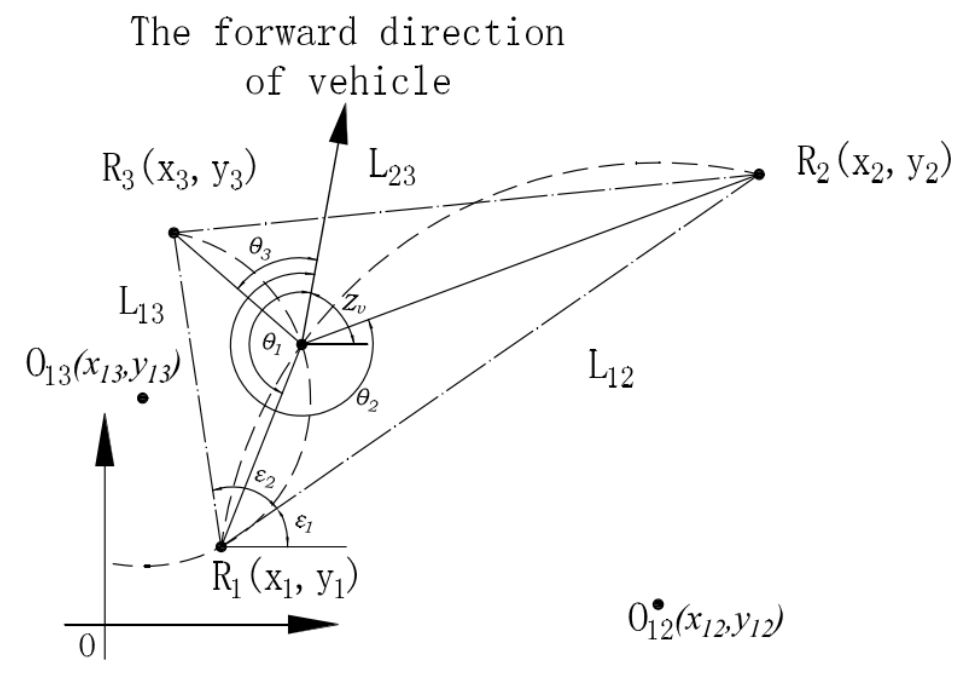

Figure 1. Relation of vehicle, three reference points and coordinate system

When the angles $\theta_{1}, \theta_{2}$ and $\theta_{3}$ between the running direction of AGV and three reference points are measured, then the coordinate $\left(x_{\mathrm{v}}, y_{\mathrm{v}}\right)$ and direction of AGV can be obtained according to the following methods.

Assume that $\alpha=\theta_{1}-\theta_{2}, \beta=\theta_{1}-\theta_{3}$, circle $O_{12}$ represents a circle which goes through three points - vehicle $V, R_{1}$ and $R_{2}$, and circle $O_{13}$ represents a circle which goes through three points - vehicle $V, R_{1}$ and $R_{3}$.Then the coordinates $O_{12}\left(x_{12}, y_{12}\right)$ and $O_{13}\left(x_{13}, y_{13}\right)$ of the centers are as follows.

$$
\begin{aligned}
& \left(x_{12}, y_{12}\right)=\left(x_{1}+P_{1}, y_{1}+Q_{1}\right), \\
& \left(x_{13}, y_{13}\right)=\left(x_{1}+P_{2}, y_{1}+Q_{2}\right),
\end{aligned}
$$

In formula (1) and formula (2),

$$
\begin{gathered}
\left(P_{1}, Q_{1}\right)=\left[\frac{L_{12} \sin \left(\alpha-\varepsilon_{1}\right)}{2 \sin \alpha}, \frac{L_{12} \cos \left(\alpha-\varepsilon_{1}\right)}{2 \sin \alpha}\right] \\
\left(P_{2}, Q_{2}\right)=\left[\frac{L_{13} \sin \left(\alpha+\varepsilon_{1}+\varepsilon_{2}\right)}{2 \sin \beta}, \frac{-L_{13} \cos \left(\alpha+\varepsilon_{1}+\varepsilon_{2}\right)}{2 \sin \beta}\right] .
\end{gathered}
$$

The coordinates $\left(x_{1}, y_{1}\right),\left(x_{2}, y_{2}\right)$ and $\left(x_{3}, y_{3}\right)$ are known, then we can calculate values of $L_{12}$, $L_{13}, \varepsilon_{1}$ and $\varepsilon_{2}$. Among them, $L_{12}$ indicates the distance between $R_{1}$ and $R_{2}, L_{13}$ indicates the distance between $R_{1}$ and $R_{3}, \varepsilon_{1}$ represents the angle between $L_{12}$ and the $x$ axis, $\varepsilon_{2}$ represents the angle between $L_{12}$ and $L_{13}$.

Via the equation (1) and (2), circle $O_{12}\left(x_{12}, y_{12}\right)$ and $O_{13}\left(x_{13}, y_{13}\right)$ can be represented: 


$$
\begin{aligned}
& O_{12}:\left(x_{\mathrm{v}}-x_{1}-P_{1}\right)^{2}+\left(y_{\mathrm{v}}-y_{1}-Q_{1}\right)^{2}=P_{1}^{2}+Q_{1}^{2}, \\
& O_{13}:\left(x_{\mathrm{v}}-x_{1}-P_{2}\right)^{2}+\left(y_{\mathrm{v}}-y_{1}-Q_{2}\right)^{2}=P_{2}^{2}+Q_{2}^{2},
\end{aligned}
$$

By solving equation (3) and (4), the location of the car can be obtained.

$$
\left(x_{\mathrm{v}}, y_{\mathrm{v}}\right)=\left[x_{1}+k\left(Q_{2}-Q_{1}\right), y_{1}-k\left(P_{2}-P_{1}\right)\right],
$$

In formula (5),

$$
k=\frac{2\left(P_{1} Q_{2}-P_{2} Q_{1}\right)}{\left(P_{2}-P_{1}\right)^{2}+\left(Q_{2}-Q_{1}\right)^{2}} .
$$

After get the position, the direction of AGV can be obtained by formula (6).

$$
z_{\mathrm{v}}=\pi+\tan ^{-1} \frac{y_{\mathrm{v}}-y_{1}}{x_{\mathrm{v}}-x_{1}}-\theta_{1} z_{\mathrm{v}}
$$

\section{TRACKING GUIDANCE}

Efficient way to guide $\mathrm{AGV}$ can reduce the track deviation in the process of transportation, improve the accuracy of the position of the loading and unloading of goods, and reduce the time of adjusting the body position at the loading and unloading point, improving transport efficiency. This article takes the path tracking method to complete tracking guidance.

The so-called tracking guidance is that the preset trajectory of AGV is divided into $\mathrm{N}$ reference points, and AGV always run to track the reference point not far ahead. This reference point is always in front of the AGV and not far away, but AGV can never catch up it. Through the arrangement of these series of reference points on the path, the running track of $A G V$ is guided, and an control objective of $\mathrm{AGV}$ is the expected reference point not far away in front of it. Running direction of AGV can be adjusted periodically by tracking guidance method, and reduce errors generated during operation ${ }^{[9]}$. Kinematics model of single steering wheel AGV is analyzed as follows.

Single steering wheel AGV is a common structure form of AGV, its gear train part includes 1 steering wheel and 2 fixed driven wheels, and it also can be attached to multiple universal wheels. Among them, the layout mode and the relative position of the steering wheel and fixed wheels determine the kinematics model of AGV, and universal support wheels have no effect on kinematics model ${ }^{[10]}$. For the single wheel AGV, a kinematics model is built, its task is solving the angle of AGV from the known position to next reference point in real-time according to known conditions such as sensor return values, in order to realize the AGV path tracking. 


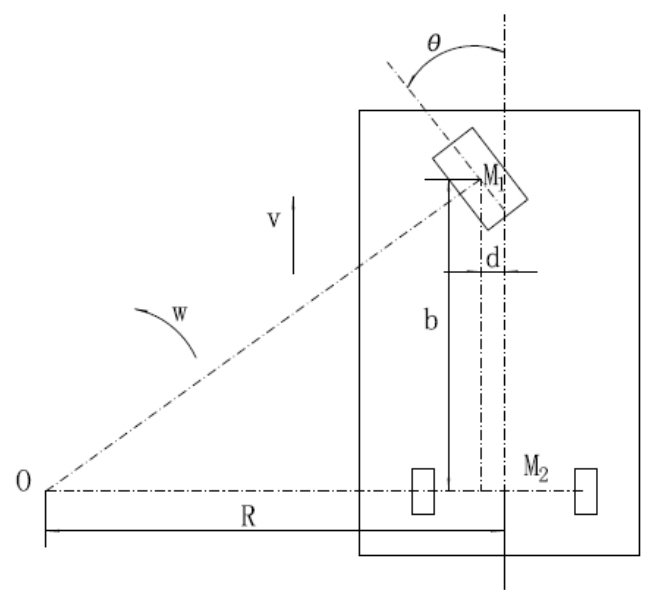

Figure 2. Layout schematic diagram of gear train of single steering wheel AGV

Layout schematic diagram of gear train of single wheel AGV is shown in Figure 2, wherein $M_{1}$ is the rotation center of the steering wheel, $M_{2}$ is the geometric center of the driven wheel, $b$ is wheelbase of AGV, $d$ is the offset of steering wheel in the direction of the center line(We call its left direction plus). When the rotation angle of steering wheel is $\theta$ (We call counterclockwise plus), $O$ is the intersection of the axis of front and rear wheels and the transient rotation center of $\mathrm{AGV}$, and $R$ is the instantaneous radius of gyration. Suppose that the current linear velocity of walking AGV is $v$ and the angular velocity is $\omega$. Since, in practice, the situation of $\theta$ is absolutely equal to zero does not exist,we just need consider the situation of $\theta \neq 0$, and follows can be obtained.

$$
\begin{aligned}
& \omega=\left\{\begin{array}{cc}
(v \cdot \sin \theta) / b & (\theta>0) \\
-(v \cdot \sin \theta) / b & (\theta<0)
\end{array}\right. \\
& \mathrm{R}=\left\{\begin{array}{cc}
b / \tan \theta^{+d} & (\theta>0) \\
-b / \tan \theta^{-d} & (\theta<0)
\end{array}\right.
\end{aligned}
$$

Posture of AGV is defined as $(x, y, z)$, Wherein, $\mathrm{x}, \mathrm{y}$ are coordinates of the geometric center of driven wheel(the $M_{2}$ in Figure 2) in the two-dimensional orthogonal coordinate of the work area, and $\mathrm{Z}$ is the attitude of $\mathrm{AGV}$ (we stipulate that $\mathrm{X}$-axis in the negative direction is 0 and the counterclockwise direction is positive). Suppose for a minimum period of time $\Delta t$, the center of driven wheel of $\mathrm{AGV}$ moves from $\mathrm{A}$ to $\mathrm{B}$, and posture of $\mathrm{A}$ is $\left(x_{\mathrm{v}}, y_{\mathrm{v}}, z_{\mathrm{v}}\right)$, posture of $\mathrm{B}$ is $\left(x_{\mathrm{b}}, y_{\mathrm{b}}, z_{\mathrm{b}}\right)$. So,

$$
\begin{aligned}
& x_{\mathrm{b}}= \begin{cases}x_{\mathrm{v}}-R \cdot \sin z_{\mathrm{v}}+R \cdot \sin \left(z_{\mathrm{v}}+\omega \cdot \Delta t\right) & (\theta>0) \\
x_{\mathrm{v}}+R \cdot \sin z_{\mathrm{v}}-R \cdot \sin \left(z_{\mathrm{v}}-\omega \cdot \Delta t\right) & (\theta<0)\end{cases} \\
& y_{\mathrm{b}}= \begin{cases}y_{\mathrm{v}}+R \cdot \cos z_{\mathrm{v}}-R \cdot \cos \left(z_{\mathrm{v}}+\omega \cdot \Delta t\right) & (\theta>0) \\
y_{\mathrm{v}}-R \cdot \cos z_{\mathrm{v}}+R \cos \left(z_{\mathrm{v}}-\omega \cdot \Delta t\right) & (\theta<0)\end{cases}
\end{aligned}
$$




$$
z_{\mathrm{b}}= \begin{cases}z_{\mathrm{v}}+\omega \cdot \Delta t & (\theta>0) \\ z_{\mathrm{v}}-\omega \cdot \Delta t & (\theta<0)\end{cases}
$$

Plug formula (7) and formula (8) into (9), then,

$$
\theta=\sqrt{\frac{\mathrm{b}}{x_{\mathrm{v}}-x_{\mathrm{b}} /\left(\sin z_{\mathrm{v}}-\sin z_{\mathrm{b}}\right)}-d}
$$

Form formula (10), We can know that $\theta$ is independent of the speed $v, \mathrm{~b}$ and $\mathrm{d}$ are the structural parameters of $\mathrm{AGV}$, the current posture $\left(x_{\mathrm{v}}, y_{\mathrm{v}}, z_{\mathrm{v}}\right)$ is measured by laser positioning technology, posture of reference point $\left(x_{\mathrm{b}}, y_{\mathrm{b}}, z_{\mathrm{b}}\right)$ is the known posture in the process of path tracking, so they are all known, then $\theta$ can be obtained easily. After a period of program running $(\Delta t)$, the running direction of AGV turns the angle $\theta$, after adjustments, AGV moves toward the direction of target point with small errors, achieving tracking guidance.

\section{EXPERIMENTAL VERIFICATION}

During the experiment, the surroundings are known, Test positioning accuracy of AGV which uses laser positioning technology during the path tracing process. Due to large body, many parts and components(all parts will produce vibration while working), and the laser positioning is more sensitive to changes in the position, a slight vibration will affect the positioning of the laser scanner, making that data of AGV position shows continuous beating in mm body, which is inconvenient for reading and affect the test results, so, we choose the method of averaging the testing values of repeated measurements in the experiment process to obtain the actual coordinates of arrival point.

$\mathrm{XOY}$ coordinate system is established within the AGV's work area, for the experiment of location and tracking which is used to verify the feasibility and stability of the study.

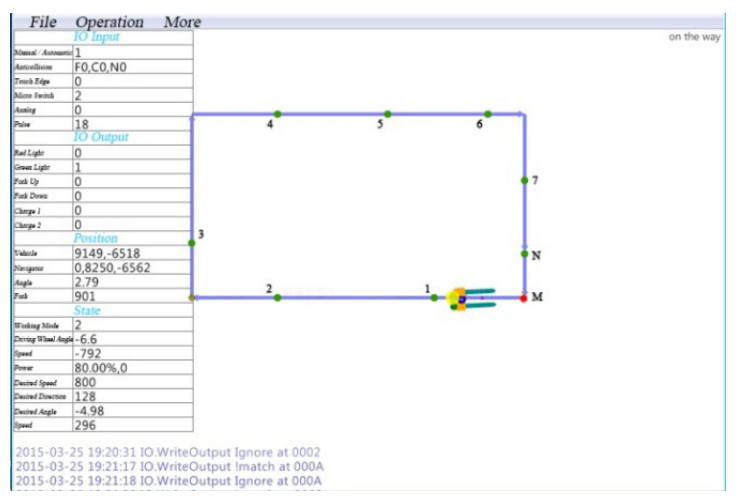

Figure 3. Experiments of tracking guidance

As shown in Figure 3, starting pose of path of AGV is M $(11000,-6500,0)$, and end position is $\mathrm{N}$ (10976, -3995,90.32), during the experiment, path sites of AGV has a total of 8 from 1 to $\mathrm{N}$, and travel path of the AGV is $\mathrm{M} \rightarrow 1 \rightarrow 2 \rightarrow 3 \rightarrow 4 \rightarrow 5 \rightarrow 6 \rightarrow 7 \rightarrow \mathrm{N}$, the 8 path sites are as shown in Table 1. Obtain the coordinates of AGV in the absolute coordinate system through the 
laser positioning technology, and compare with the actual coordinates, then we can get errors, and the final results of the experiment are shown in table 1.

Table 1 shows theory posture $\left(x_{\mathrm{v}}, y_{\mathrm{v}}, z_{\mathrm{v}}\right)$ of each point on the path and the actual pose $\left(x_{\mathrm{v}}^{\prime}, y_{\mathrm{v}}^{\prime}, z_{\mathrm{v}}^{\prime}\right)$ of AGV during tracking guidance process. Analysis data shows that the position error is less than $\pm 10 \mathrm{~mm}$, and the angle error is in $\pm 0.3^{\circ}$. Experiments show that, by the method of laser positioning and path tracing above, AGV can successfully complete the given task, and verify the feasibility of the research, as the error is in the allowable range and meet the performance requirements of AGV industry.

Table 1. experimental results of tracking guidance

\begin{tabular}{cccc}
\hline $\begin{array}{c}\text { Serial } \\
\text { number }\end{array}$ & $\begin{array}{c}\text { Target point } \\
\text { coordinates }\left(\mathrm{mm}, \mathrm{mm}{ }^{\circ}\right)\end{array}$ & $\begin{array}{c}\text { Actual arrival } \\
\text { point }\left(\mathrm{mm}, \mathrm{mm},{ }^{\circ}\right)\end{array}$ & Error $\left(\mathrm{mm}, \mathrm{mm},{ }^{\circ}\right)$ \\
\hline 1 & $(7510,-6503,358.90)$ & $(7508.7,-6509.3,359.07)$ & $(1.3,6.3,0.17)$ \\
2 & $(1008,-6495,0.28)$ & $(1004.7,-6490,0.31)$ & $(3.3,5,0.03)$ \\
3 & $(-3505,-3014,268.63)$ & $(-3509.3,3012.6,268.92)$ & $(4.3,1.4,0.29)$ \\
4 & $(988,1489,179.66)$ & $(988.7,1489.3,179.92)$ & $(0.7,0,0.26)$ \\
5 & $(4988,1484,180.42)$ & $(4991.3,1484,180.33)$ & $(3.3,0,0.09)$ \\
6 & $(9985,1513,178.30)$ & $(9992.3,1514.3,178.21)$ & $(7.3,1,0.09)$ \\
7 & $(11020,-1680,88.85)$ & $(11014.7,-1689.3,88.45)$ & $(5.3,9.3,0.40)$ \\
$\mathrm{N}$ & $(10976,-3995,90.32)$ & $(10982,-3988,90.46)$ & $(6,7,0.14)$ \\
\hline
\end{tabular}

\section{REFERENCES}

[1] Shi Enxiu,Huang Yumei.Study on Positioning Method of Autonomous Navigation Vehicle AGV.Journal of transducer technology,2007,01:233-236.

[2] Fang Qiang, Xie Cunxi.Autonomous Positioning Navigation of Mobile Robot Based on Vision[J]. Machine Tool \& Hydraulics, 2004, (17): 40-56.

[3] Ni Zhen. Research on the key technology of laser guidance of four wheel differential omnidirectional mobile AGV [D].Chongqing University,2013.

[4] Liu Yang. Discussion on laser positioning algorithm of laser guidance AGV[J].Logistics Technology and Application,2007,11:100-101.

[5] Teng Yunlong, Shi Yibing. A three-dimensional positioning method based on three satellites[J].Journal of Central South University,2012,12:3449-3453.

[6] A New Method of Global Path Planning for AGV[J]. International Journal of Plant Engineering and Management,2006,01:51-58.

[7] Yuan Wei, Sun Jie, Cao Zuoliang, Tian Jing, Yang Ming. A combined object-tracking algorithm for omni-directional vision-based AGV navigation[J]. Optoelectronics Letters,2010,02:137-139.

[8] S.Butdee,A.Suebsomran, F.Vignat, P.K.D.V.Yarlagadda.Control and pathprediction of an Automate Guided Vehicle[J].Journal of Achievements in Materials and Manufacturing Engineering.2008,2(3); P 70-75

[9] Mehdi Yahyaei, J.E.Jam\&R.Hosnavi.Controlling the navigation of automatic guided vehiele (AGV) using integrated fuzzy logic controllerwith programmable logic controller (IFLPLC)IJ8. Int J Ady Manuf Technol.2010.47:795-807.

[10] Yao Jiajia. Vision-based Road Detection and Navigation for AGV Systems[D].Zhejiang University, 2014. 
International Journal of Computer Science \& Engineering Survey (IJCSES) Vol.6, No.3, June 2015

\section{Author}

Cheng Jing was born on March 25, 1990, in Xianning City, Hubei Province, China. She received the college diploma in mechanical design, manufacturing and automation from the China Three Gorges University, Yichang City, China, in2013.Then, She was admitted as a graduate student of Shanghai University of Engineering Science, Shanghai, China, in2013.

She is now a second-year grad student and majors in robotics and intelligent control. Her interests focus mainly in the area of robot, embedded systems, and intelligent control.

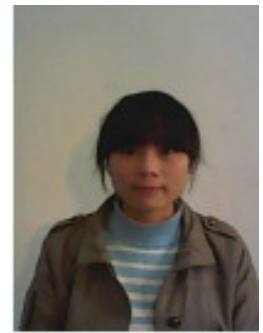

